NASA Technical Memorandum 100547

\title{
MACH NUMBER EFFECTS ON TRANSONIC AEROELASTIC FORCES AND FLUTTER CHARACTERISTICS
}

(NASA-T 1005 47) MACH NUHEEE EFEECTS ON

TEANSCNIC AEECEIASTIC FORCES AND FLUTTER

CHARACTERISTICS (NASA) $13 \mathrm{p}$ CSCL 01A

Unclas

G3/02 0123407

ROSS W. MOHR

JOHN T. BATINA

HENRY T. Y. YANG

FEBRUARY 1988

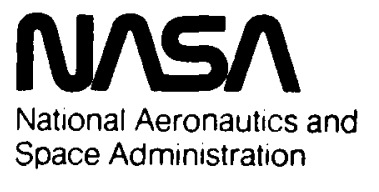

Langley Research Center

Hampton, Virginia 23665-5225 


\author{
Ross W. Mohr \\ Purdue University \\ West Lafayette, Indiana 47907 \\ John T. Batina \\ NASA Langley Research Center \\ Hampton, Virginia 23665-5225 \\ Henry T. Y. Yang \\ Purdue University \\ West Lafayette, Indiana 47907
}

\begin{abstract}
Transonic aeroelastic stability analyses and flutter calculations are presented for a generic transpontype wing based on the use of the CAP.TSD (C.omputational Aeroelasticity Program - Iransonic Smail Disturbance) finite-difference code. The CAP.TSD code has been recently developed for transonic unsteady aerodynamic and aeroelastic analysis of complete aircratt configurations. In this study, a binary aeroelastic system consisting of simple bending and torsion modes was used to study aeroelastic behavior at transonic speeds. Generalized aerodynamic forces are presented for a wide range of Mach number and reduced frequency. Aeroelastic characteristics are presented for variations in freestream Mach number, mass ratio, and bending-torsion frequency ratio. Flutter boundaries are presented which have two transonic dips in flutter speed. The first dip is the "usual" transonic dip involving a bending-dominated flutter mode. The second dip is characterized by a single degree-of-freedom torsion oscillation. These aeroelastic results are physically interpreted and shown to be related to the steady-state shock location and changes in generalized aerodynamic forces due to freestream Mach number.
\end{abstract}

\section{Nemenclature}

$A_{i j}$

generalized aerodynamic force resulting from pressure induced by mode $\mathrm{j}$ acting through displacements of mode $i$

AR full-span aspect ratio

b wing root semichord, $c_{\gamma} / 2$

c wing local chord

$\mathrm{c}_{r} \quad$ wing reference chord (root chord)

$C_{p} \quad$ pressure coefficient

critical pressure coefficient

$f(x, y) \quad$ structural mode shape

$k \quad$ reduced frequency, $\omega c_{r} / 2 U$

$M \quad$ freestream Mach number

$q(t) \quad$ pulso motion

qo pulse amplitude

Q nondimensional dynamic pressure,

$\left(U /\left(b \omega_{\alpha} \sqrt{\mu}\right)\right)^{2}$

Q nondimensional dynamic pressure at flutter

s Laplace Iransform variable, $\sigma+i \omega$

$t$ time, nondimensionalized by freestream speed and wing reference chord

tc nondimensional time at the pulse center

TR wing taper ratio

U freestream speed

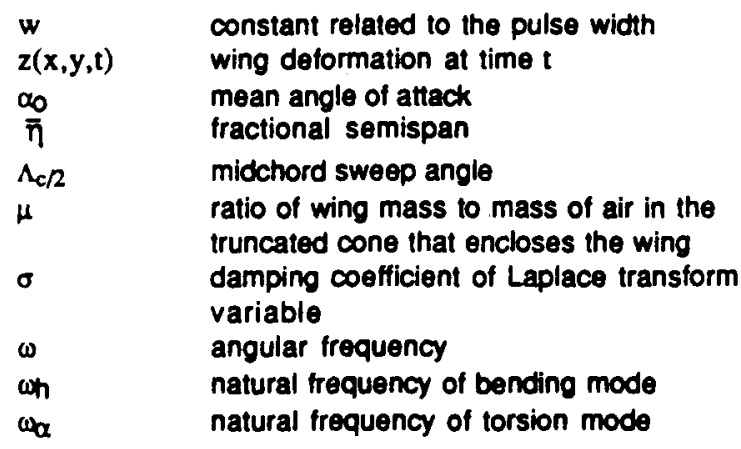

\section{Introduatlon}

Prediction of Iransonic unsteady aerodynamics for aeroelastic analysis has been aided by the development of finite-difference computer codes. An example of one of the most fully developed finite-difference codes for aeroelastic analysis is the CAP.TSD 1 code recently developed at the NASA Langley Research Center. The name CAP.TSD is an acronym for Compulational Aeroelasticity Rrogram. Iransonic Small Disturbance. The code permits the calculation of steady and unsteady flows about complete aircraft configurations for aeroelastic applications in the transonic speed range. Steady and unsteady pressure comparisons were presented in Refs. 1 to 3 for numerous cases which demonstrated the geometrical applicability of CAP.TSD. These calculated results were generally in good agreement with available experimental pressure data which validated CAP.TSD for multiple component applications with mutual aerodynamic interference effects. Preliminary flutter applications of CAP.TSD were presented in Ref. 4 for a simple well-defined wing case. The calculated flutter boundaries compared well with the experimental data which gives confidence in CAP.TSD for aeroelastic prediction. More recently, the code was modified to include entropy and vorticity effects to treat cases with relatively strong shock waves.5 Without these effects, the isentropic formulation typically overpredicts the shock strength and locates the shock too far aft in comparison with experiment. The modified code includes the entropy and vorticity effects while retaining the relative simplicity and cost efficiency of the small-disturbance potential formulation. As shown in Ref. 5, the results obtained by including these effects were in very good agreement with parallel Euler calculations for attached-flow strong-shock cases.

In Refs. 1 to 5 the CAP.TSD code was shown to be accurate and efficient for transonic unsteady aerodynamic and aeroelastic applications. It is therefore timely to utilize such a computer code to study the generalized aerodynamic forces (GAFs) and aeroolastic stability characteristics of wings in Iransonic flow. The purpose of the paper is therefore to report on the results of a transonic unsteady 
aerodynamic and aeroelastic analysis of a generic transportlype wing. The primary objectives of the research were to: (1) calculate transonic GAFs and aeroelastic characteristics for a wide range of parameter variations; and (2) correlate and physically interpret the resulting pressure distributions, GAFs, and aeroelastic stability characteristics including flutter.

In this study, a binary aroelastic system consisting of simple bending and torsion modes was used to investigate aeroelastic behavior at transonic speeds. Results were obtained for incremental changes in freestream Mach number. Effects of mass ratio and bending-torsion frequency ratio on aeroelastic characteristics were also considered. The present work is analogous to the numerous aeroelastic studies performed for Iwo-dimensional airfoils by many researchers including 1sogai, 6 Yang et al., 7 and Edwards, et al.8 In Refs. 6 to 8, aeroelastic analyses were performed for a large range of treestream conditions and structural parameter variations for numerous airfoil sections. These studies contributed significantly to the understanding of aeroelastic behavior at transonic Mach numbers. The present work may similarly give physical insight into the flow mechanisms which control transonic aeroelastic behavior of three-dimensional wings.

The motivations for the study were to gain a better understanding of transonic unsteady flows and aeroelastic behavior, and the need to develop efficient computational methods to predict and investigate aeroelastic phenomena. For example, the transonic GAFs in the present study were obtained by extending the pulse transfer-function analysis of Refs. 9 and 10 to treat flexible modes of motion. In this analysis, the GAFs are obtained indirectly from the response due to a smoothly varying exponentially shaped pulse. Use of the pulse iransfer-function analysis gives considerable delail in the frequency domain with a significant reduction in cost over the alternative method of forced harmonic oscillation. The paper presents a description of the pulse analysis including results and comparisons which assess the capability. Transonic aeroolastic stability analyses are performed with the GAFs from the pulse analysis, using state-space aeroelastic modeling such as that reported in Refs. 11 to 14. The stability analysis of the present study is an extension of the state-space model of Refs. 13 and 14 for binary aeroelastic analysis of a three-dimensional wing. A brief description of the procedure is given in a subsequent section.

A secondary objective of the present work was to determine whether the analysis tectniques are capable of predicting more challenging aeroelastic problems of significant current interest. For example, the results to be presented show a second, more critical (lower) dip in flutter speed at transonic Mach numbers slightly higher than the first dip. A flutter boundary with two transonic dips has indeed been found experimentally for a transport-lype supercritical-wing flutter model tested at the NLR in the Netherlands. 15 The first dip was identified in Ref. 15 as the "usual" transonic dip which involves a bending-dominated flutter mode, for the wing in predominantly attached flow. The second dip. however, occurred in a range of Mach number where the flow was separated and was identified as a torsional-buzz type of flutter, since the flutter mode was almost identical with the torsion vibration mode shape. The phenomenon was attributed in Refs. 15 and 1610 an aerodynamic resonance which occurred in a narrow range of Mach number at higher angles of attack for this wing. It has been suggested by Mabey, 17 that if this aerodynamic resonance occurs at frequencies close to wing torsional frequencies, the single degree-of-freedom torsional buzz may result. A similar torsional buzz phenomenon has also been reported by Moss and Pierce 18 where the primary torsion mode of a series of wings tended to be strongly excited under separated flow conditions. This excitation led to a sustained high-amplitude response or limit-cycle torsional buzz. In this case, a strong shock wave occurred on the upper surface of the wing. which was approximately parallel to the torsion-mode node line. Also, the shock was of fairly uniform strength across the span in the outboard region of the wing. The movement of the shock with angle of attack was such that the slope of the local pitching moment about the node line was negative rather than positive. As discussed in Refs. 17 and 18, these features seem to be necessary conditions for the occurrence of torsional buzz.

Although the current work is limited to inviscid attached flow, the results to be presented exhibil similar flutter behavior as observed in the experiments reported in Refs. 15 to 18 and described above. Certainly when strong shocks are present in the inviscid calculations or when the physical situation is known to involve separated flow, the present analysis needs to be extended to account for unmodeled effects. Nevertheless, the detalled comparisons and physical interpretations presented may give an increased understanding of unsteady transonic flows and insight into the fundamental aeroelastic mechanisms responsible for flutter at transonic speeds.

\section{Computational Procedures}

In this section, the computational procedures used in the present study are described including the CAP.TSD code, the pulse transfer-function analysis, the structural mode shapes, and the aeroelastic stability analysis.

\section{CAP-TSD Code}

The recently developed CAP.TSD 1 finite-difference code was used to calculate the steady and unsteady aerodynamics presented in the paper. The CAP.TSO code is an unsteady transonic small-disturbance (TSD) code developed for transonic aeroelastic analysis of complete aircraft configurations. The code uses a time-accurate approximate factorization (AF) algorithm developed by Batina19 for efficient solution of the unsteady TSD equation. The AF algorithm consists of a Newton linearization procedure coupled with an internal iteration technique. The CAP.TSD code is capable of treating combinations of lifting surfaces and bodies, and includes the following algorithm features: (1) Engquist-Osher monotone differencing, (2) nonreflecting lar field boundary conditions, (3) secondorder accurate spatial differencing in supersonic regions of the flow, and (4) entropy and vorticity effects to treat cases with strong shock waves. Further details of the algorithm development and solution procedures are reported in Refs. 3 , 5, and 19. A detailed description of CAP.TSD may be found in Ref. 1.

\section{Pulse Tranafer-Functlon Anolyals}

Generalized aerodynamic forces which are typically used in aeroelastic analyses may be obtained by calculating several cycles of a harmonically forced oscillation with the determination of the forces based upon the last cycle of oscillation. The method of harmonic oscillation requires one flow field calculation for each value of reduced frequency of interest. By contrast, GAFs may be determined for a wide range of reduced frequency in a single flow field calculation by the pulse transfer-function analysis.9,10 in the pulse analysis, the GAFs are computed indirectly from the response of the flow field due to a smoothly varying exponentially shaped pulse. Results computed using the pulse analysis for a pitching flat plate airfoil were first presented in Rel. 9. These results were in good agreement with parallel linear theory calculations which validated the 
accuracy of the analysis. Applications to transonic airfoil cases were also in good agreement with GAFs computed using the harmonic method. Calculations for a flat plate wing undergoing a simple rigid pitching motion were presented in Ref. 10. These three-dimensional results were also shown to agree well with linear theory for reduced frequencies less than unity. Therefore, because of the computational efficiency of the pulse transfer-function analysis, the capability was extended in the present study to treat general flexible modes such as the natural vibration modes of a wing. The pulse is expressed as

$$
q(t)=q_{0} e^{-w\left(t-t_{c}\right)^{2}}
$$

where $q_{0}$ is the pulse amplifude, $w$ is a constant related to the width of the pulse, and $t_{c}$ is the time at the center of the pulse. The deformation of any point on the wing at time $t$, is determined by the product of the pulse motion and the mode shape. The deformation $z(x, y, t)$ is then given by

$$
z(x, y, t)=q_{0} e^{-w\left(t-t_{c}\right)^{2}} f(x, y)
$$

where $f(x, y)$ is the mode shape, and $x$ and $y$ are nondimensional Cartesian coordinates in the streamwise and spanwise directions, respectively. A small pulse is prescribed in a given mode of motion and the aerodynamic transients are calculated. The aerodynamic transients are then used to obtain the GAFs in the frequency domain by a transfer-function analysis. In this analysis, a fast Fourier transform (FFT) of the aerodynamic transients is divided by the FFT of the corresponding pulse motion to calculate the GAFs. The GAFs, $A_{i j}$, are defined as the integrated forces resulting from pressure induced by mode $j$ acting through the displacements of mode $i$. The transform assumes that the system is linear which is a valid approximation for most transonic cases, since experience has shown that the response for harmonic or aeroelastic motion is, in general, locally linear for small amplitudes of oscillation.9-14

\section{Structural Mode Shapes}

In this investigation, simple polynomial equations were assumed to describe the vibration mode shapes. The following equations simulate first bending and first torsion mode shapes of a wing, respectively:

$$
\begin{gathered}
f_{B}=y\left[\left(x-\frac{1}{2}\right) \sin \Lambda_{c / 2}+y \cos \Lambda_{c / 2}\right] \\
f_{T}=y\left[\left(x-\frac{1}{2}\right) \cos \Lambda_{c / 2}-y \sin \Lambda_{c / 2}\right]
\end{gathered}
$$

where $\Lambda_{c / 2}$ is the midchord sweep angle of the wing. The equation describing the mode shape due 10 bending (Eq. (3)) assumes that a node line is perpendicular to the wing midchord line at the root. The equation describing the mode shape due to torsion (Eq. (4)) assumes that a node line coincides with the wing midchord line. The amplitude of the pulse motion is selected to give a maximum tip deflection due to bending of approximately four percent of the semispan and a maximum angle of attack at the tip due to torsion of one degree.

\section{Anroglatle Stabllity Analyses}

Constant sectional properties from root to tip are assumed for the aeroelastic analyses. This simplified modeling is consistent with the assumed mode shapes of Eqs. (3) and (4), which in turn assumes that the aeroelastic motion of the wing can be described by a linear combination of the fundamental bending and torsion modes. The calculations were performed using state-space aeroelastic modeling such as that reported in Refs. 11 to 14. Edwards, et al.11 used a state-space model employing Pade' approximants to model the unsteady airloads and demonstrated good agreement with a time-marching technique for a linearized case. Bland and Edwards 12 demonstrated that such locally linear procedures may be used with airloads derived from a transonic smalldisturbance $\operatorname{cod} \theta$ for airfoil applications. Batina and Yang 13,14 used a similar procedure to study transonic aeroelastic stability and response behavior of airfoils with active controls. The stability analysis of the present study is an extension of the state-space model of Refs. 13 and 14 for binary aeroelastic analysis of a three-dimensional wing. The resulting locally linear stability model provides a relatively inexpensive determination of aeroelastic $\therefore$ stability, while retaining the nonlinear properties of the transonic mean flow. The model is derived by assuming a linear superposition of airloads due to bending and torsion motions. The required airloads are approximated by curvefitting the CAP-TSD GAFs with a Pade' approximating function. 13 The function may then be rewritten as a set of ordinary differential equations which, when coupled to the equations of motion and Laplace transformed, leads to a linear first-order matrix equation

$$
\frac{s}{\omega_{\alpha}}\{z)=[A](z)
$$

where $\{z\}$ contains the displacements, velocities, and augmented states and [A] is a real matrix of constant elements. Equation (5) is solved using linear eigenvalue solution techniques for specified values of the nondimensional dynamic pressure $Q$, defined as $\left(U /\left(b \omega_{\alpha} \sqrt{\mu}\right)\right)^{2}$. The resulting eigenvalues are plotted in a dynamic pressure "root-locus" type format in the complex s-plane.

\section{Besults and Discussion}

Results are first presented from the pulse transferfunction analysis to assess the accuracy and efficiency of the capability by making comparisons with other calculations. The pulse analysis was then used in a parameler study to investigate the effects of freestream Mach number on GAFs at transonic speeds. Results are also presented from the aeroelastic stability analysis, obtained using the GAFs from the pulse analysis.

\section{Conflauration Analyzed}

The wing selected for the present study is the Royal Aircraft Establishment (RAE) research wing " $A$ " that was tested in the RAE $8 \mathrm{ft} . \times 6 \mathrm{ft}$. transonic wind tunnel.20 The wing is a structurally rigid model that has a simple planform without dihedral or twist and a symmetric RAE 101 airfoit section with a maximum thickness-to-chord ratio of $9 \%$. As shown in Fig. 1, the wing has a midchord sweep angle of $30^{\circ}$, a taper ratio of one-third, and a fullspan aspect ratio of six. The polynomial mode shapes that were assumed for the wing are shown in Fig. 2. The RAE 
wing $A$ is a standard configuration selected by the AGARD Fluid Dynamics Panel for steady flow calculations. Although the wing is a rigid model and no flutter data is available, the wing was selected for the present study because of the simplicity of the planform and the availability of experimental steady pressure data.20 The experimental steady pressure data that is reported in Ref. 20 , however, was measured with the wing mounted on an axisymmetric fuselage. Therefore, a simple fuselage was modeled with CAP.TSD, along with the wing, to allow for direct comparison with the steady pressure data. All of the other calculations to be presented were performed without the fuselage.

Calculations were performed for thirteen values of freestream Mach number including $M=0.8,0.825,0.85$ $0.875,0.9,0.91,0.92,0.93,0.94,0.95,0.96,0.97$, and 0.98 . Results are presented mainly for a subset of these cases, namely at $M=0.8,0.9,0.92,0.94,0.96$, and 0.98 . For $M \geq 0.94$, relatively strong shock waves are present in the flow. For these cases, the isentropic catculation typically overpredicts the shock strength and locates the

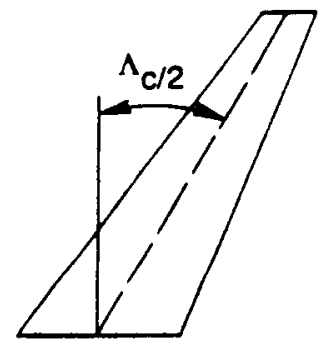

RAE wing "A"

$$
\Lambda_{c / 2}=30^{\circ}, A R=6.0, T R=1 / 3
$$

Fig. 1 RAE wing " $A$ " geometry.

Mode 1: Bending

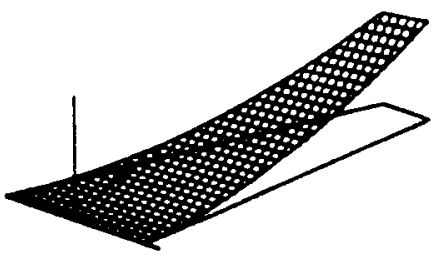

Mode 2: Torsion

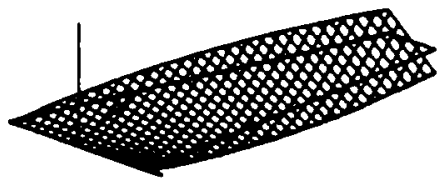

Fig. 2 Structural mode shapes. shock too far aft in comparison with the physical situation. Therefore, the calculations were repeated for $M \geq 0.94$ by including the entropy and vorticity effects to accurately treat these cases. Furthermore, calculations were also performed at $M=0.9$ for a series of wings created by varying the midchord sweep angle from $\Lambda_{\mathrm{c} / 2}=14^{\circ} 1040^{\circ}$. These results were obtained to determine effects of wing sweep on transonic GAFs and flutter. These results may be found in Ref. 21.

\section{Steady Presaure Reaults}

Steady pressure results are presented first 10 assess the basic character of the transonic flow. Since the unsteady flow field and hence generalized aerodynamic forces depend strongly on the transonic steady-state flow, the steady pressure distributions frequently can give physical insight into critical flow mechanisms which control aeroelastic phenomena. Of interest are the flow characteristics in the wing tip region such as the shock strength and shock location relative to the node line of the torsion mode.

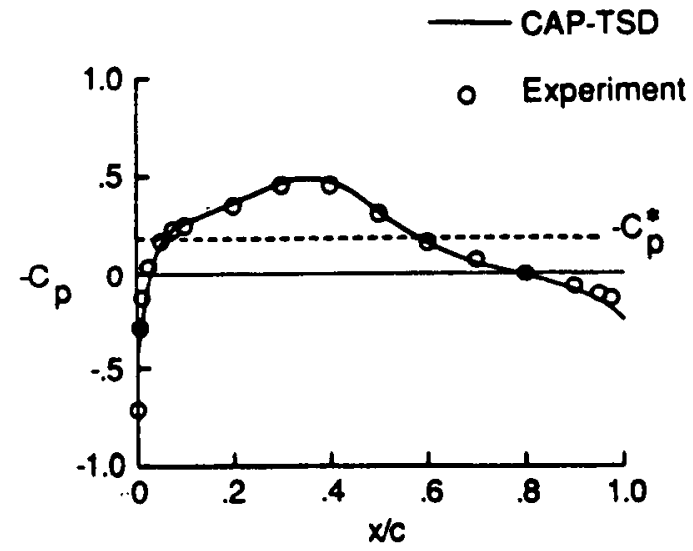

(a) $\bar{\eta}=0.40$.

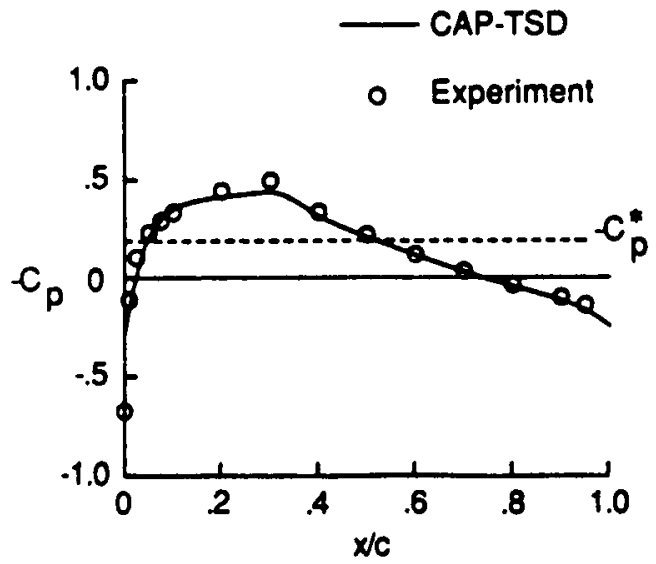

(b) $\bar{\eta}=0.85$

Fig. 3 Comparison between CAP.TSD and experimental steady pressure distributions on the RAE wing " $A$ " at $M=0.90$ and $\alpha_{0}=0^{\circ}$. 


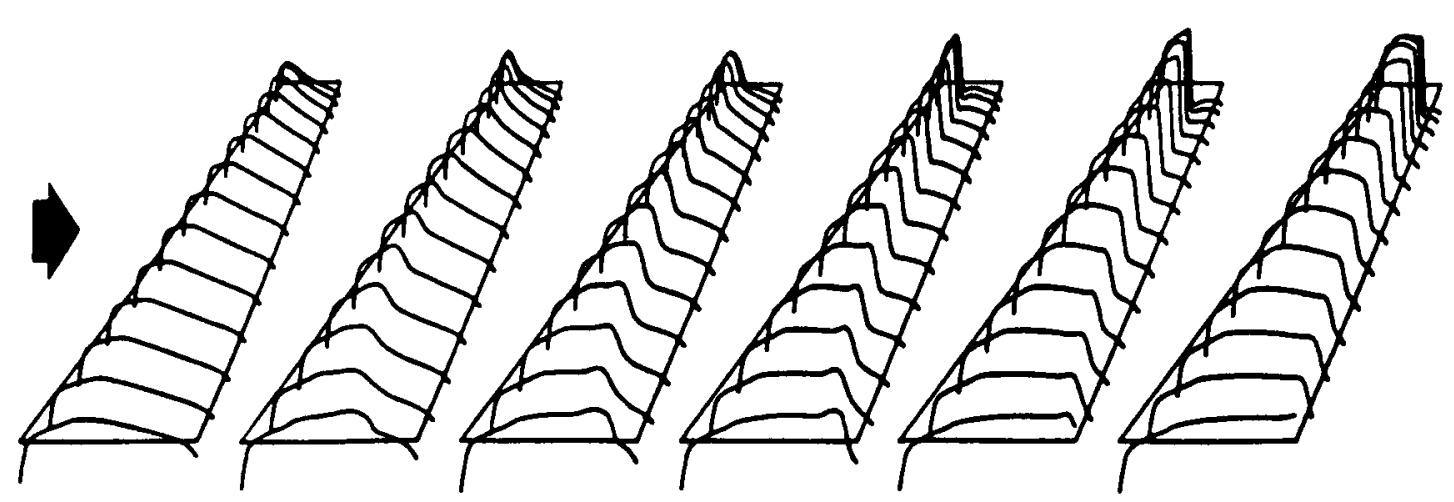

Fig. 4 Sleady pressure distributions for a range of Mach number at $\alpha_{0}=0^{\circ}$.

Experimental Date Comparison. - To assess the accuracy of the CAP-TSD solution for this configuration, results were obtained for the RAE wing $A$ and fuselage at $M=0.9$ and $\alpha_{0}=0^{\circ}$ for comparison with the experimental data. Steady pressure distributions at two span stations along the wing are presented in Fig. 3. Results for $\bar{\eta}=0.40$ are shown in Fig. 3(a); results for $\bar{\eta}=0.85$ are shown in Fig. 3(b). The calculated pressures are identical along the upper and lower wing surfaces, since the airfoil section is symmetric and the wing is at $0^{\circ}$ angle of attack. These pressure distributions indicate the presence of embedded supersonic regions along the upper and lower surfaces. A mild shock wave also occurs in the inboard region of the wing (not shown). In general, the CAP.TSD pressures agree well with the experimental data.

Effecte of Frentrenm Mach Number. - Steady pressure distributions at six different Mach numbers and $\alpha_{0}=0^{\circ}$ are presented in Fig. 4. The pressures for $M \geq 0.94$ were computed including the entropy and vorticity corrections. At $M=0.8$, the calculated pressures indicate that the flow is entirely subcritical. At $M=0.9$, there is a

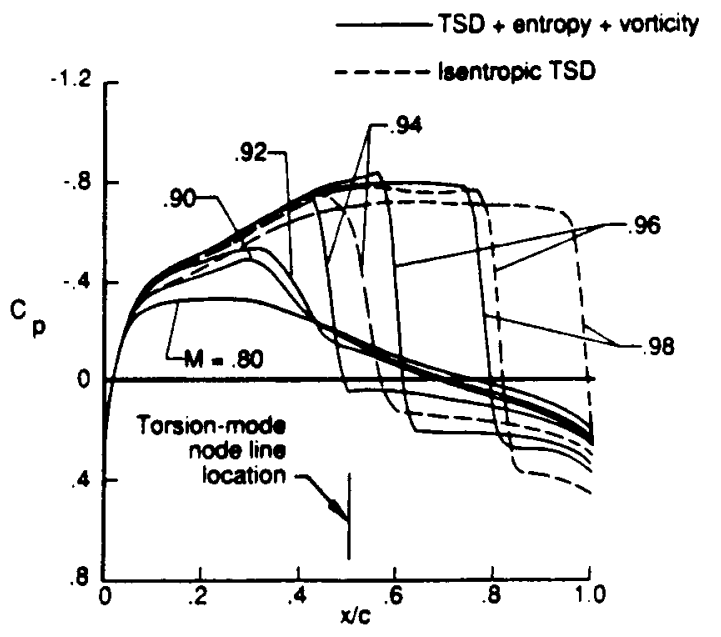

Fig. 5 Steady pressure distributions at $\bar{\eta}=0.9$ for a range of Mach number at $\alpha_{0}=0^{\circ}$. mild shock wave in the inboard region of the wing that weakens in the outboard direction toward the wing tip. When the freestream Mach number was increased to $M=0.92$ and 0.94, a moderately strong shock wave forms across the span along the upper and lower wing surfaces. In the outboard region of the wing, the shock is approximately parallel to the torsion-mode node line at the local midchord. Here the shock is also of fairly uniform strength in the spanwise direction. These features of the shock location and strength are similar to those reported in Rets. 17 and 18, which were considered to be necessary (but not sufficient) conditions for torsional buzz to occur. When the Mach number was further increased to $M=0.96$ and 0.98 , the shock moves aft towards the trailing edge. Since the steady pressure distributions show relatively small changes in the range $0.8 \leq M \leq 0.9$, it is anticipated that only small changes will occur in the GAFs. Between $M=0.92$ and 0.98 , much larger changes in the GAFs are expected because of the larger differences in the steady flows, especially in the wing tip region.

Figure 5 shows more detailed comparisons of the steady pressure distributions at $\bar{\eta}=0.9$ for the six Mach numbers ranging from $M=0.8$ to 0.98 . These comparisons of pressures clearly show the transonic steady flow characteristics in the wing tip region. For $M \geq 0.94$, results corresponding to the calculations with and without entropy and vorticity effects are shown. Without entropy and vorticity effects, the shock is stronger and moves aft much faster with increasing Mach number. At $M=0.96$ and 0.98 , for example. the isentropic shock is located approximately $20 \%$ chord downstream of the nonisentropic shock. Also, at $M=0.98$ the isentropic calculation predicts that the shock wave is at the trailing edge. Furthermore, the pressure distributions of Fig. 5 show that the shock wave crosses the torsion-mode node line with increasing Mach number near $M=0.94$. This fact is expected to play a significant role in the aeroelastic behavior of the wing.

\section{Generalleed Ancodynamic Force Resulte}

Generalized aerodynamic forces are presented beginning with an assessment of the accuracy and elficiency of the pulse iransfer-function analysis relative to harmonic oscillation calculations. Further results are then presented from the calculations which include the nonisentropic corrections to demonstrate the effects of freestream Mach number on GAFs. All of the results are presented in the form of real and imaginary components of the GAFs, $A_{i j}$, as functions of the reduced frequency $k$. In the present study, bending and torsion are defined as modes 1 and 2 , respectively. 
Whene Theop compatien. - To assess the accuracy of the pulse transfer-function analysis to treat flexible mode shapes, results were oblained for the wing modeled as a flat plate at $M=0.9$ and $\alpha_{0}=0^{\circ}$. These results are compared with linear theory results computed using RHOIV, an assumed pressure mode kernel function method for simple harmonic motion.22.23 The RHOIV generalized aerodynamic forces were obtained for eleven values of reduced frequency ranging from $k=0.0$ to 1.0 in increments of 0.1 . Comparisons of results from the pulse analysis and RHOIV are given in Fig. 6. The results shown are for the bending mode GAF due to torsion motion, $A_{12}$, which is similar 10 wing unsteady lift-curve slope. The two sets of results are in good agreement, for the wide range of $k$ shown, which validates the puise analysis for application 10 flexible mode shapes. Results for the other three GAFs show similar good agreement between CAP.TSD and RHOIV and therelore are not shown.21

Iransonle Comparieon. - To assess the accuracy of the pulse analysis for transonic applications, GAFs were obtained for the wing at $M=0.9$ and $\alpha_{0}=0^{\circ}$. The accuracy of these forces is determined by making comparisons with similar results computed using the harmonic method. Results from the harmonic method were obtained for eleven values of reduced frequency ranging from $k=0.0$ to 1.0 in increments of 0.1 . Comparisons of the $A_{12}$ GAF calculated by the pulse analysis and the harmonic method are shown in Fig. 7. The two sets of results are in very good agreement which validates the pulse transfer-function analysis for application to transonic cases. Results for the other three GAFs show similar good agreement between the pulse analysis and the harmonic method and therefore are not shown.21

With respect to computational efficiency, the harmonic method required one flow field calculation for each value of reduced frequency for each mode of motion. This is in contrast to the pulse analysis which determined the GAFs for the entire range of frequency in a single flow field

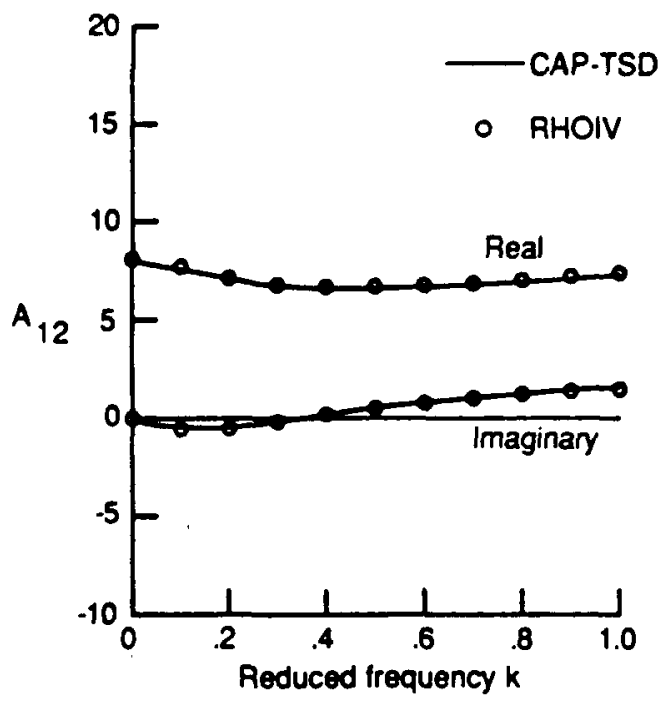

Fig. 6 Comparison between generalized aerodynamic forces calculated by CAP-TSD (linear) and RHOIV for the wing modeled as a flat plate at $M=0.9$ and $\alpha_{0}=0^{\circ}$. calculation for each mode. Since eleven values of reduced frequency were selected for calculation by the harmonic method, the computational cost of obtaining the pulse results was consequently about an order of magnitude less than the cost of the harmonic method results. For the case considered here, the pulse analysis is therefore a relatively inexpensive method of determining transonic GAFs which in turn makes it economically feasible to conduct the parameler variation study reported below.

Effects of Erenatrenm Mach Number. - To determine the effects of freestream Mach number on the GAFs, results are presented for the wing at $M=0.8,0.9$, $0.92,0.94,0.96$, and 0.98 at zero mean angle of attack, corresponding to the steady pressure distributions presented in Figs. 4 and 5. Comparisons of GAFs at these Mach numbers are presented in Figs. 8(a) to 8 (d).

For the GAFs resulting from the pressure induced by bending ( $A_{11}$ and $A_{21}$ shown in Figs. 8(a) and $8(b)$, respectively), the real part of $A_{21}$ shows the most significant change due to Mach number. With increasing Mach number, the real part of A21 decreases in magnitude for $0.92 \leq M<0.96$. This decrease is attributed to an aft shift in the aerodynamic center which begins to occur after $M=0.92$ due to the formation of the shock wave in the tip region. As shown in the steady pressure distributions of Figs. 4 and 5 , there is a mild shock wave at $M=0.92$ which grows in strength when the Mach number is increased to $M=0.94$. Since $A_{21}$ is similar to a wing moment coefficient and the torsion-mode node line is located at the local midchord, an att shift in the aerodynamic center produces a decrease in magnitude of the real part of $A_{21}$. Significant changes in aeroelastic behavior may occur for $M>0.92$ because of this large decrease in magnitude of $A_{21}$.

For the GAFs resulting from the pressure induced by torsion ( $A_{12}$ and $A_{22}$ shown in Figs. $8(c)$ and $8(d)$. respectively), increasing the Mach number produced large changes in the forces especially in the low $k$ range. These

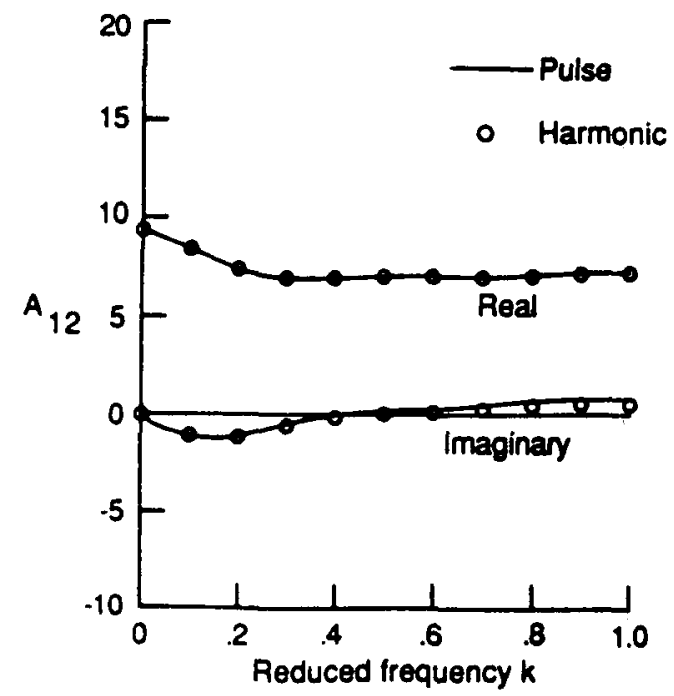

Fig. 7 Comparison belween CAP.TSD generalized aerodynamic forces calculated by pulse analysis and harmonic analysis for the wing at $M=0.9$ and $\alpha_{0}=0^{\circ}$. 


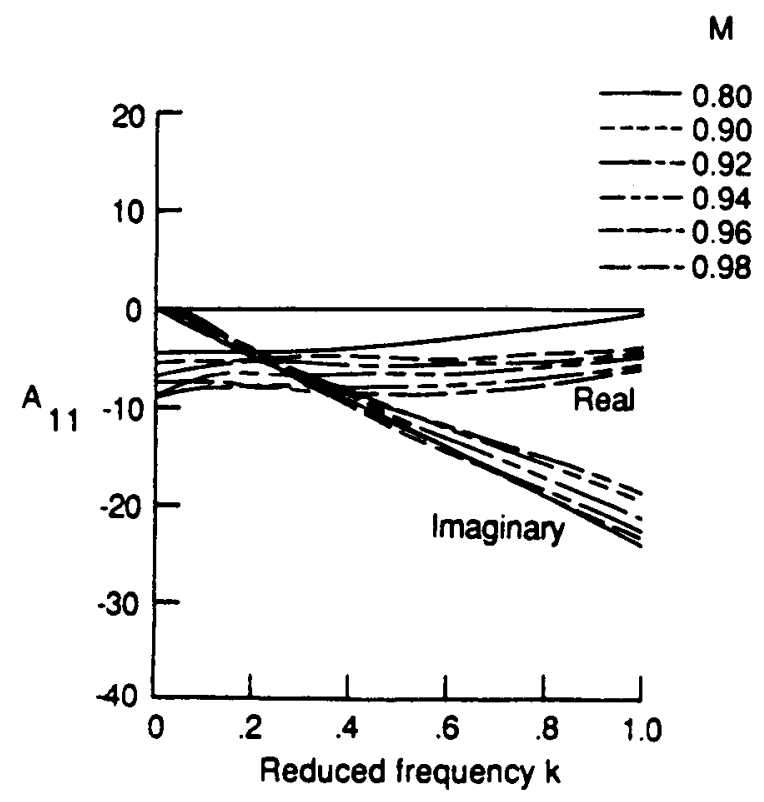

(a) GAF resulting from pressures due 10 bending weighted by bending displacements.

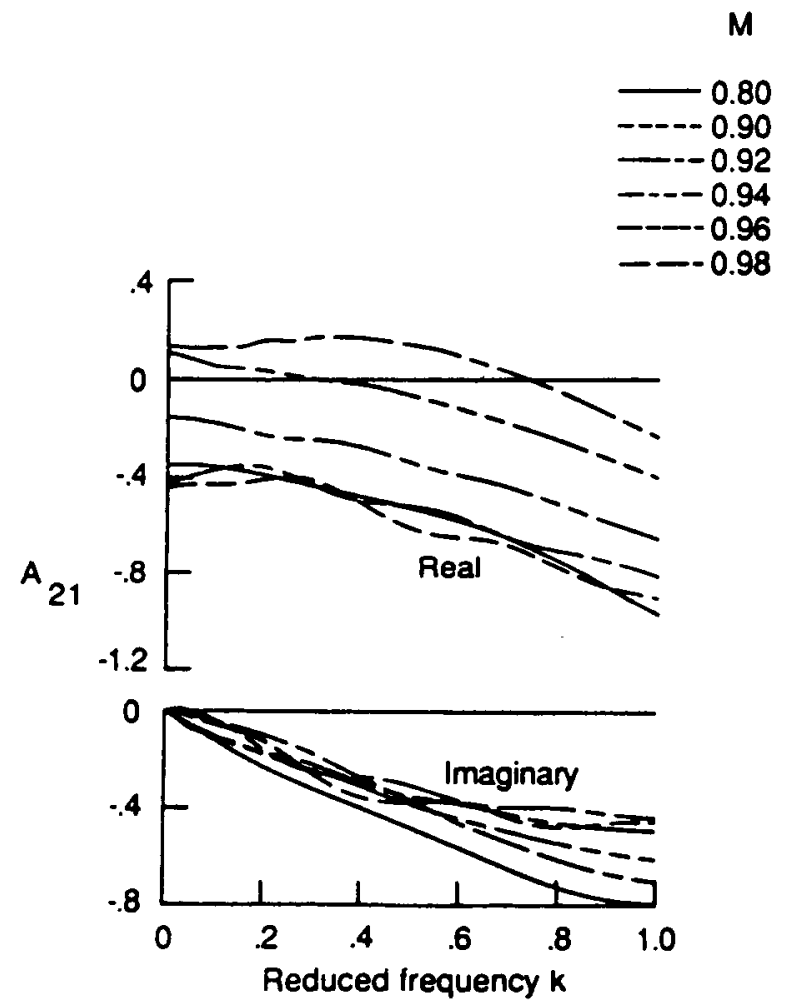

(b) GAF resulting from pressures due to bending weighted by torsion displacements.

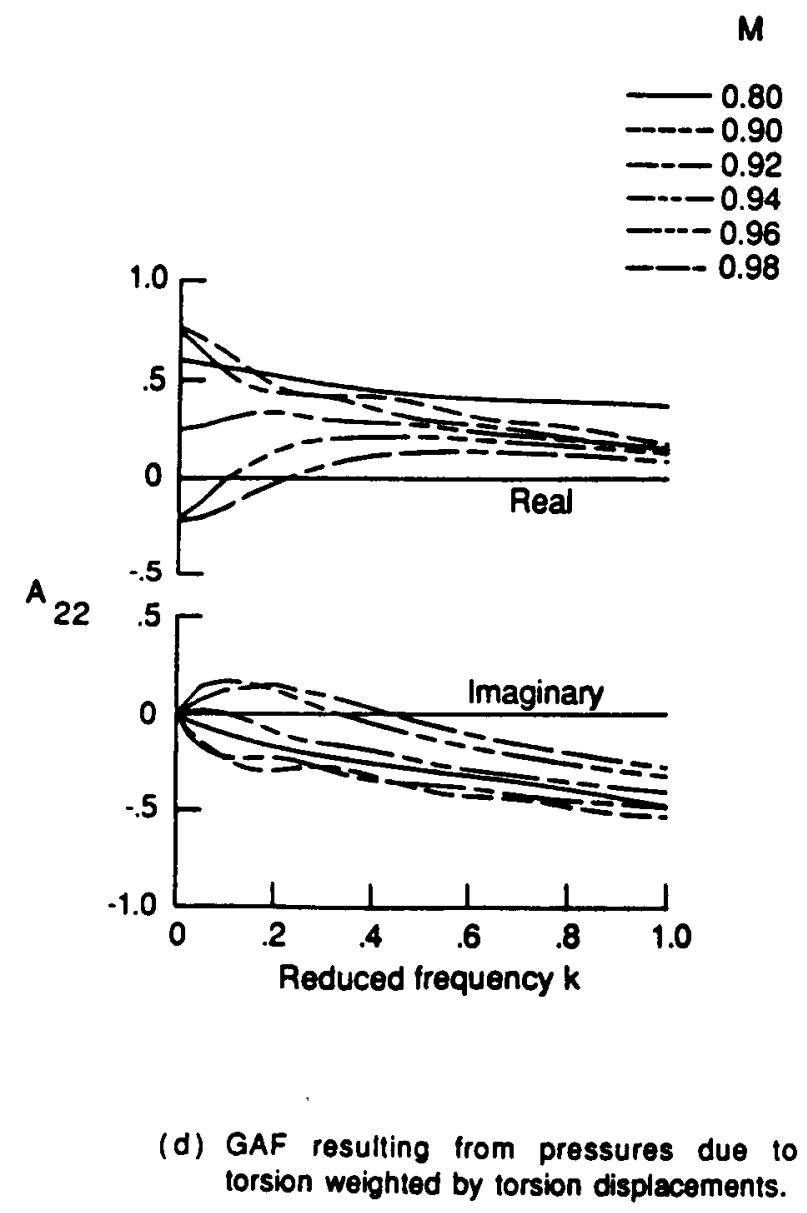

Fig. 8 Comparison of generalized aerodynamic forces

(GAFs) for a range of Mach number at $\alpha_{0}=0^{\circ}$. 
changes in GAFs are of primary interest because they occur in the range of reduced frequency where flutter typically occurs $(0.1<k<0.4)$. With increased Mach number from $M=0.8$ to 0.92 , for example, both the real and the imaginary parts of $A_{12}$ increase in magnitude. The $A_{12}$ force is similar 10 wing lift-cune slope which increases as Mach number increases. This increases both the magnitude and the phase lag of $A_{12}$ which in lurn controls the aeroelastic behavior for $0.8 \leq M \leq 0.92$. As discussed by Isogai6 and later by Zwann,24 it is the increased phase lag of A 12 which is the flow mechanism that produces the transonic dip in flutter speed. Also with increased Mach number, the real part of $A_{22}$ generally decreases which is again attributable to an aft shift in aerodynamic center due to shock waves. Furthermore, for the higher Mach numbers in the range $0.94 \leq M \leq 0.98$, the imaginary part of $A_{2} 2$ changes sign for low reduced frequencies. This change in

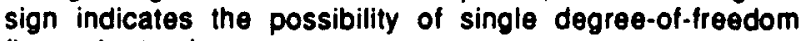
flutter in torsion.

\section{Aeroelastic Sinblitiy and Flutter Reaulte}

Aeroelastic stability and flutter results are presented next to investigate aeroelastic characteristics at transonic speeds. Calculations were pertormed for several values of freestream Mach number, mass ratio, and for $\omega_{h} / \omega_{\alpha}=0.1$, 0.3, and 0.5. Root-loci plots for $\mu=100$ and $\omega_{h} / \omega_{\alpha}=0.3$ are presented in Fig. 9 for the six Mach numbers corresponding to the steady pressure distributions of Figs. 4 and 5 and the GAFs of Figs. 8(a) to 8(d). Stability results for $M=0.8,0.9,0.92,0.94,0.96$, and 0.98 are shown in Figs. 9 (a) through $9(f)$, respectively, computed including the nonisentropic corrections. The stability plots show the migration of bending and torsion root-loci as Mach number is increased. The increment in dynamic pressure shown in the figures is $\Delta Q=0.2$. Al $M=0.8$, the curves indicate a classical bending-torsion type of aeroelastic behavior. Here the torsion branch moves to the left in the stable lett-half

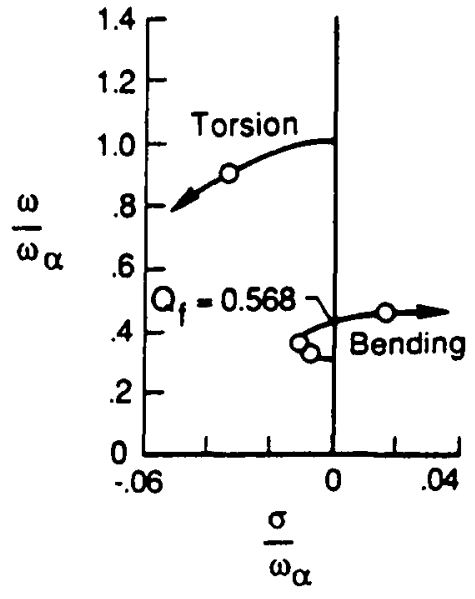

(a) $M=0.80$.

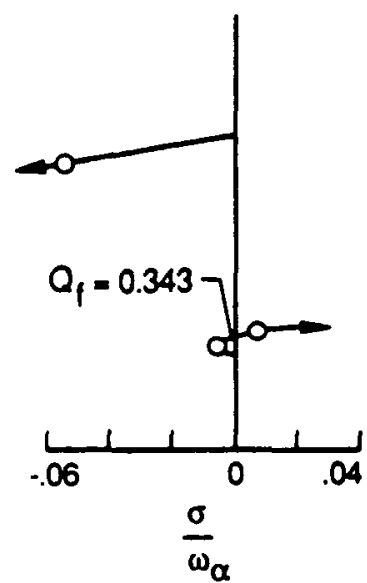

(b) $M=0.90$.

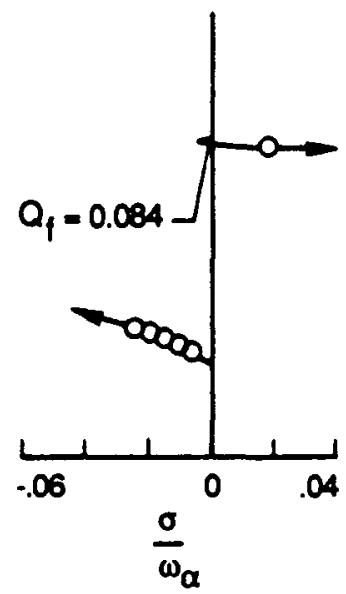

(e) $\mathrm{M}=0.96$.

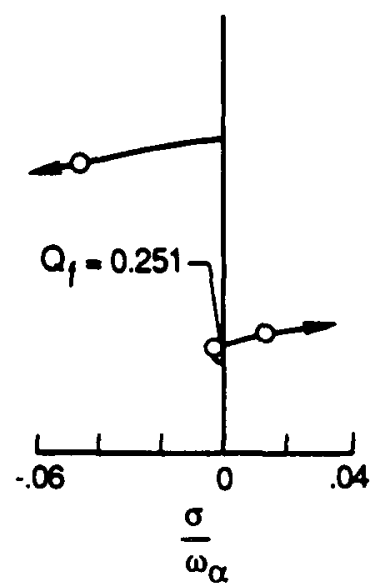

(c) $M=0.92$.

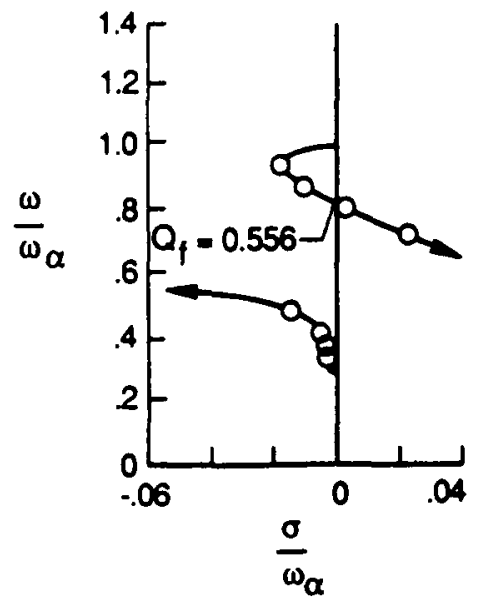

(d) $M=0.94$. (f) $M=0.98$

Fig. 9 Nondimensional dynamic pressure root-loci for a range of Mach number at $\alpha_{0}=0^{\circ}, \mu=100, \omega h / \omega \alpha=0.3$, and $\Delta Q=0.2$. 
plane, with increasing dynamic pressure, while the bending-dominated branch becomes the flutter mode. With increasing Mach number in the range $0.8 \leq M \leq 0.92$, the flutter dynamic pressure decreases and flutter occurs at a lower frequency. Between $M=0.92$ and 0.94 , the root-loci coalesce in frequency and then swilch, in that the flutter crossings at $M=0.94,0.96$, and 0.98 originate from the wind-off torsion mode rather than the bending mode. A rapid increase in the flutter frequency thus occurs in the range $0.92 \leq M \leq 0.96$, from a value close to the bending natural frequency at $M=0.92$ to a value close to the torsion natural frequency at $M=0.96$. These aeroelastic characteristics are further explained by considering the corresponding flutter boundaries which are given in Fig. 10, and correlating these results with the steady pressures of Figs. 4 and 5 , and the GAFs of Figs. 8(a) to 8(d). Figure 10(a) shows the flutter

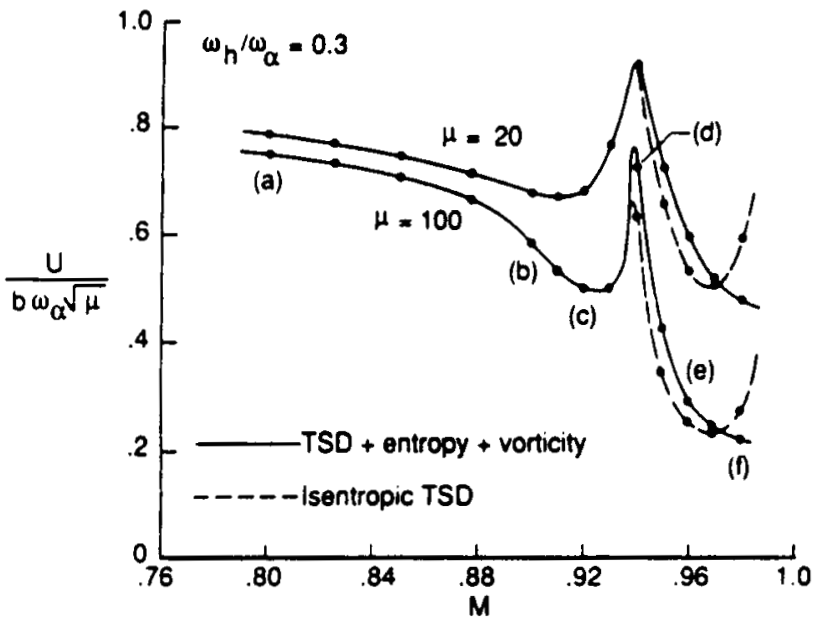

(a) flutter speed index versus Mach number.

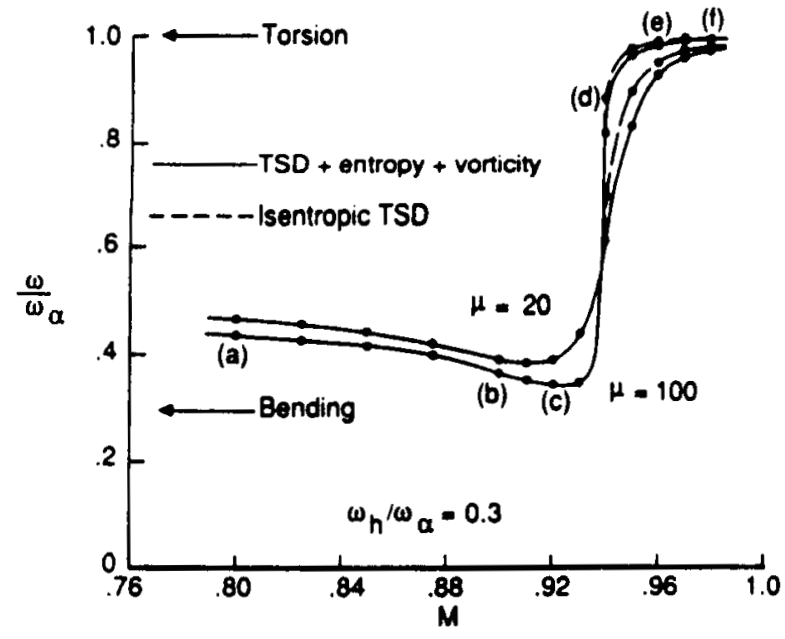

(b) nondimensional flutter frequency versus Mach number.

Fig. 10 Effects of mass ratio on flutter characteristics at $\alpha_{0}=0^{\circ}$. speed index (defined as $U /\left(b \omega_{\alpha} \sqrt{\mu}\right)$ ) versus Mach number and Fig. 10(b) shows the nondimensional flutter frequency (defined as $\omega / \omega_{\alpha}$ ) versus Mach number. In these figures. the points along the $\mu=100$ curves which are labeled (a) through (1), correspond to the root-loci of Figs. 9(a) 10 $9(\eta)$, respectively. Both isentropic as well as nonisentropic curves are plotted. Flutter results are also shown in Fig. 10 for $\mu=20$ to indicate effects due to mass ratio.

The flutter boundaries of Fig. 10(a) show two dips in flutter speed index, the second of which is deeper than the first. These transonic dips in flutter speed are deeper at the higher mass ratio of $\mu=100$. More specifically, as Mach number increases in the range $0.8 \leq M \leq 0.92$, the flutter speed decreases as expected. This decrease is due to the

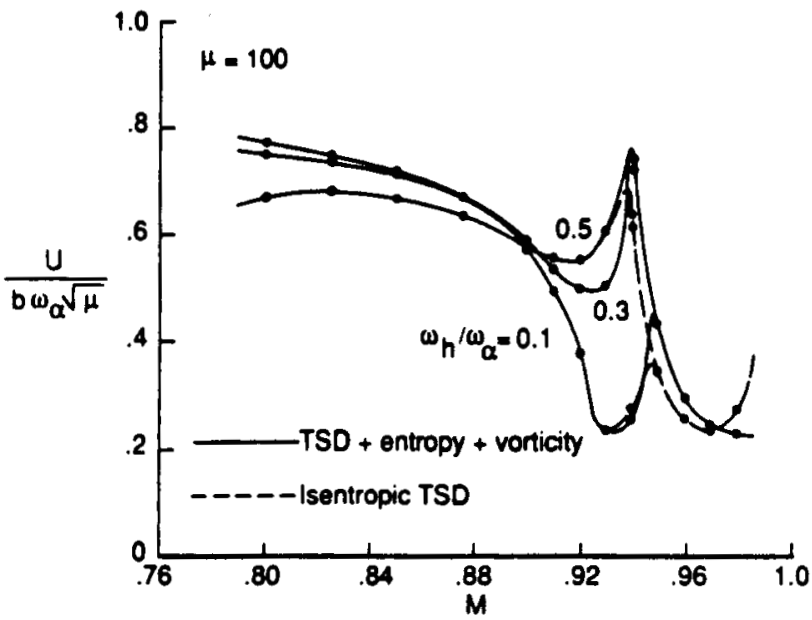

(a) flutter speed index versus Mach number.

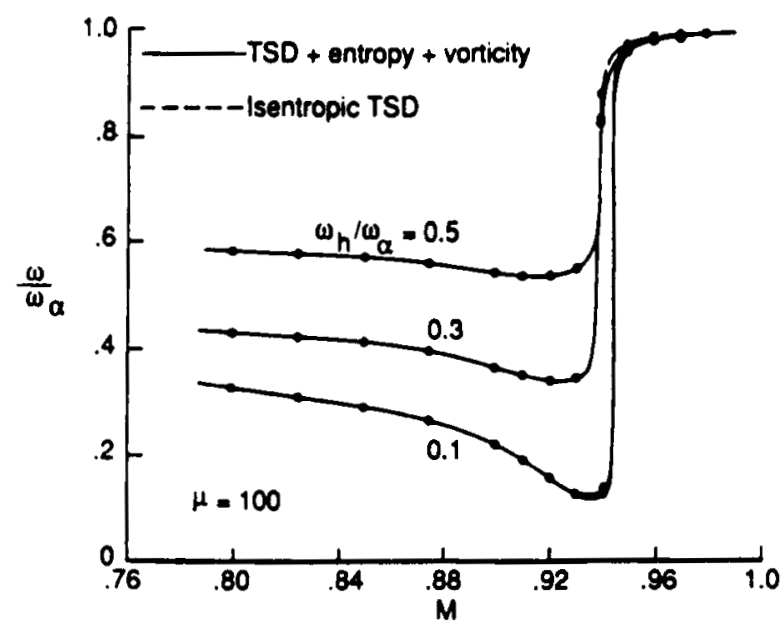

(b) nondimensional flutter frequency versus Mach number.

Fig. 11 Effects of bending-torsion frequency ratio on flutter characteristics at $\alpha_{0}=0^{\circ}$. 
increased phase lag of the $A_{12}$ generalized aerodynamic force which occurs with increasing Mach number (Fig. 8(c)). The bottom of the first dip occurs at $M=0.91-0.92$ for both $\mu=20$ and 100 . Here the shocks are relatively weak, and the frequency curves of Fig. 10(b) suggest that the flutter mode is predominately bending since the flutter frequency is close to the bending mode trequency. Inspection of the corresponding fitter eigenvectors confirmed this. At $M=0.92$ and $\mu=100$, for example, the ratio of the magnitudes of bending and torsion in the flutter mode eigenvector is 8:1. With a further small increase in Mach number, there is a rapid increase in flutter speed (Fig. 10(a)) which defines the "other side" of the first dip. Physically this occurs because there is a significant decrease in the magnitude of the $A_{2} 1$ and $A_{2} 2$ GAFs for $0.92<M<0.94$ (Figs. 8 (b) and 8 (d)) due to the aft shift in aerodynamic center. Recalling that these GAFs are like moment coefficients, they decrease in magnitude when the aerodynamic center shifts aft toward the torsion-mode node line. Associated with this change in GAFs is a significant decrease in the magnitude of the imaginary part of $A_{22}$ which indicates a loss of aerodynamic damping in torsion. The decrease in torsional damping causes the flutter mode to rapidly transition from a bending-dominated instability at $M=0.92$ to a torsion-dominated instability at $M=0.94$. This transition is shown by the rapid increase in flutter frequency from $M=0.92$ to 0.94 shown in Fig. $10(\mathrm{~b})$. It was also shown in Figs. $9(c)$ and $9(d)$, by the switch in root-loci, which results in a much higher frequency at the flutter crossing. Near $M=0.94$, the shock, which is approximately parallel to the torsion-mode node line in the outboard region of the wing. crosses the node line with increasing Mach number. This has a destabilizing effect with respect to flutter since the damping in torsion is actually negative for low values of $k$ as shown in Fig. 8(d). The flutter speeds in the first part of the second dip are lower in the isentropic calculations since the shock wave is stronger and located further aft of the torsion-mode node line, as previously shown in Fig. 5. The further loss of torsional damping at the higher Mach numbers produces a flutter mode that is almost identical to the torsion mode shape, as suggested by the fiutter frequency values for $M>0.95$ shown in Fig. 10(b). The ratio of the magnitudes of torsion and bending in the flutter eigenvector at $M=0.97$ and $\mu=100$, for example, is 80:1. Therefore the instability in the second dip is a single degree-ot-freedom flutter similar to the torsional-buzz phenomenon reported in Refs. 15 to 18. With a further increase in Mach number, the shock wave in the isentropic calculations moves aft to the trailing edge near $M=0.97$, which has a stabilizing influence on the aeroelastic system since the flow about the wing is mosily supersonic. With the shock at the trailing edge, the flutter speed increases rapidly thus defining the "other side" of the second flutter dip. In the more-accurate nonisentropic calculations, however, the shock wave does not reach the trailing edge for the range of freestream Mach number considered. Hence, the flutter characteristics are considerably different and the importance of including entropy and vorticity effects is evident.

Effects of bending-torsion frequency ratio on flutter characteristics are shown in Fig. 11. Flutter speed index and nondimensional flutter frequency as functions of freestream Mach number are shown in Figs. 11(a) and 11(b), respectively. Calculations were performed for $\omega h / \omega_{\alpha}=0.1,0.3$, and 0.5 with a mass ratio of $\mu=100$. In Fig. 11, the curves for $\omega_{h} / \omega_{\alpha}=0.3$ and $\mu=100$ are the same as those shown in Fig. 10. The results indicate that the first dip in flutter speed is significantly influenced by $\omega_{h} / \omega_{\alpha}$. With $\omega_{h} / \omega_{\alpha}=0.5$, for example, the dip is very shallow in comparison to the dip with $\omega_{h} / \omega_{a}=0.1$. By decreasing $\omega_{h} / \omega_{\alpha}$ from 0.5 to 0.1 , the flutter speed decreases by $56 \%$. The bottom of the dip also shifts to a slightly higher Mach number. The flutter frequency curves of Fig. 11 (b) show the same trends for the three frequency ratios, each curve being shifted toward the respective bending mode frequency for $M<0.93$. The flutier mode at the bottom of the first flutter dip. however, is somewhat different for the three cases. Inspection of the flutter eigenvectors revealed that the ratios of magnitudes of bending and torsion for $\omega_{h} / \omega_{\alpha}=0.1,0.3$, and 0.5 are $70: 1$, $8: 1$, and $5: 1$, respectively. This indicates that the flutter mode at the bottom of the dip for $\omega_{h} / \omega_{\alpha}=0.1$ is a single degree-of-freedom bending instability. The results further indicate that the second dip in flutter speed is independent of the bending-torsion frequency ratio which is not unexpected since here the flutter mode is a single degree-of-freedom torsional instability.

\section{Coneluding Remrike}

Transonic aeroelastic stability analyses and flutter calculations were presented for a generic transport-type wing based on the use of the CAP-TSD (C.omputational Aeroelasticity Rrogram - Iransonic Small Disturbance) finite-difference code. In this study, a binary aeroelastic system consisting of simple bending and torsion modes was used to study aeroelastic behavior at transonic speeds. Transonic generalized aerodynamic forces (GAFs) and aeroelastic characteristics were computed for a wide range of freestream Mach number. The CAP.TSD calculations were performed by including entropy and vorticity effects to more accurately treat cases with strong shock waves.

An efficient method of calculating transonic generalized aerodynamic forces was extended to treat general flexible modes of molion of a wing. The method presented was the pulse transfer-function analysis which determines the unsteady forces indirectly from the response due to a smoothly varying exponentially shaped pulse. Comparisons of calculated GAFs for both linear and nonlinear cases showed good agreement with alternative calculations which verified the pulse analysis for application to flexible modes. Furthermore, the computational resources required by the pulse analysis were an order of magnitude less than those required by the harmonic method.

The pulse analysis was used in a parameter study to investigate the effects of freestream Mach number on GAFs at transonic speeds. The GAFs from the pulse analysis were utilized in aeroelastic stability and flutter calculations. Flutter boundarles were computed which had two transonic dips in flutter speed. The first dip was the "usual" transonic dip which involved a bending-dominated flutter mode. The second dip was characterized by a single degree-of-freedom torsion oscillation. This single degree-of-freedom flutter occurred at the higher Mach numbers considered due to a loss of aerodynamic damping in torsion when the steady shock location was downstream of the torsion-mode node line.

\section{Acknowledament}

The work constitutes a part of the first author's M.S. thesis at Purdue University and was supported by the NASA Langley Graduate Aeronautics Program under grant NAG-1. 372 .

\section{Beferences}

1Batina, J. T.; Seidel, D. A.; Bland, S. R.; and Bennett, R. M.: "Unsteady Transonic Flow Calculations for Realistic Aircraft Configurations," AIAA Paper No. 87-0850, Presented at the AIANASME/ASCE/AHS 28th Structures, Structural Dynamics, and Materials Conference, Monterey, California, April 6-8, 1987. 
2Bennett, R. M.; Bland, S. R.; Batina, J. T.; Gibbons, M. D.; and Mabey, D. G.: "Calculation of Steady and Unsteady Pressures on Wings at Supersonic Speeds with a Transonic Small Disturbance Code," AIAA Paper No. 87-0851, Presented at the AIANASME/ASCE/AHS 28th Structures, Structural Dynamics, and Materials Conference, Monterey, California, April 6-8, 1987.

3Batina, J. T.: "Unsleady Transonic Algorithm Improvements for Realistic Aircraft Applications," AIAA Paper No. 88-0105. Presented at the AIAA 26th Aerospace Sciences Meeting. Reno, Nevada, January 11.14, 1988.

${ }^{4}$ Cunningham, H. J.: Batina, J. T.: and Bennett, R. M.: "Modern Wing Flutter Analysis by Computational Fluid Dynamics Methods," Presented at the ASME Winter Annual Meeting, Boston, Massachusetts, December 13-18, 1987.

5Batina, J. T.; Seidel, D. A.; Bennett, R. M.: Cunningham, H. J.; and Bland, S. R.: "Steady and Unsteady Transonic Small Disturbance Analysis of Realistic Aircraft Configurations," to be presented at the Transonic Symposium, NASA Langley Research Center, Hamplon, Virginia, April 19-21, 1988.

6/sogai, K.: "Numerical Study of Transonic Flutter of a Two-Dimensional Airfoil," NAL TR-617T, National Aerospace Laboratory. Tokyo, Japan, July 1980.

7Yang, T. Y.: Guruswamy, P.; and Striz, A. G.: "Application of Transonic Codes to Flutter Analysis of Conventional and Supercritical Airfoils," Journal of Aircrafl, vol. 19. March 1982, pp. 211-220.

8 Edwards, J. W.; Bennett, R. M.; Whitlow, W., Jr.; and Seidel, D. A.: "Time-Marching Transonic Flutter Solutions Including Angle-of-Attack Effects," Journal of Aircratt, vol. 20. November 1983, pp. 899-906.

9Seidel, D. A.; Bennett, R. M.; and Whitlow, W., Jr.: "An Exploratory Study of Finite Difference Grids for Transonic Unsteady Aerodynamics," AlAA Paper No. 83-0503, Presented at the AIAA 21 st Aerospace Sciences Meeting. Reno, Nevada, January 10-13, 1983.

10 Seidel, D. A.; Bennett, R. M.; and Ricketts, R. H.: "Some Recent Applications of XTRAN3S," AIAA Paper No. 83-1811, Presented at the AIAA Applied Aerodynamics Conference, Danvers, Massachusetts, July 13-15, 1983.

11 Edwards, J. W.; Bennett. R. M.; Whitlow. W., Jr.; and Seidel, D. A.: "Time Marching Transonic Flutter Solutions Including Angle of Attack Effects," AlAA Paper No. 82-3685, Presented at the AIAAASME/ASCE/AHS 23rd Structures. Structural Dynamics, and Materials Conference, New Orleans, Louisiana, May 10-12, 1982.

12Bland, S. R.; and Edwards, J. W.: "Airfoil Shape and Thickness Effects on Transonic Airloads and Flutter." Jeurnal of Alrcraft vol. 21, March 1984, pp. 209-217.
13Batina, J. T.; and Yang, T. Y.: "Application of Transonic Codes to Aeroelastic Modeling of Airfoils Including Active Controls, Journal of Aircraft, vol. 21, August 1984, pp. 623.630 .

14Batina, J. T.: and Yang, T. Y.: "Transonic Calculation of Airtoil Stability and Response with Active Controls," AIAA Paper No. 84-0873, Presented at the AIANASME/ASCE/AHS 25th Structures, Structural Dynamics, and Materials Conference, Palm Springs, California, May 14-16, 1984.

15Zwann, R. J.: "Verification of Calculation Methods for Unsteady Airloads in the Prediction of Transonic Flutter," Journal of Aircraft, vol. 22, October 1985, pp. 833-839.

16den Boer, R. G.; and Houwink, R.: "Analysis of Transonic Aerodynamic Characteristics for a Supercritical Airfoil Oscillating in Heave, Pitch and with Oscillating Flap," AGARD CP.374, January 1985.

17Mabey, D. G.: "A Review of Some Recent Research on Time-Dependent Aerodynamics," The Aeronautical Journal of the Royal Aeronautical Society, February 1984, pp. 23-37.

18Moss, G. F.; and Pierce D.: "The Dynamic Response of Wings in Torsion at High Subsonic Speeds," AGARD CP.226, 1977.

19Batina, J. T.: "An Elficient Algorithm for Solution of the Unsteady Transonic Small-Disfurbance Equation," AIAA Paper No. 87.0109. Presented at the AlAA 25th Aerospace Sciences Meeting, Reno, Nevada, January 12-15, 1987.

20Treadgold, D. A.; Jones, A. F.; and Wilson, K. H.: -Pressure Distribution Measured in the RAE 8ft. $\times 6 \mathrm{ft}$. Transonic Wind Tunnel on RAE Wing " $A$ " in Combination with an Axi-Symmetric Body at Mach Numbers of 0.4, 0.8, and 0.9," in "Experimental Data Base for Computer Program Assessment," AGARD-AR-138, May 1979.

21Mohr, R. W.: "Effects of Mach Number and Wing Sweep on Transonic Aeroelastic Forces and Flutter Characteristics," M.S. Thesis, Purdue University, West Lafayette, Indiana, December 1987.

22Rowe, W. S.: Sebastian, J. D.; and Petrarca, J. R.: "Reduction of Computer Usage Costs in Predicting Unsteady Aerodynamic Loadings Caused by Control Surface MotionsAnalysis and Results," NASA CR-3009, March 1979.

23Petrarca, J. R.; Harrison, B. A.; Redman, M. C.; and Rowe, W. S.: "Reduction of Computer Usage Costs in Predicting Unsteady Aerodynamic Loadings Caused by Control Surface Motions-Computer Program Description," NASA CR.145354, June 1978.

24 Zwann, R. J.: "Aeroelastic Problems of Wings in Transonic Flow," NLA MP 81005 U, February 1981. 


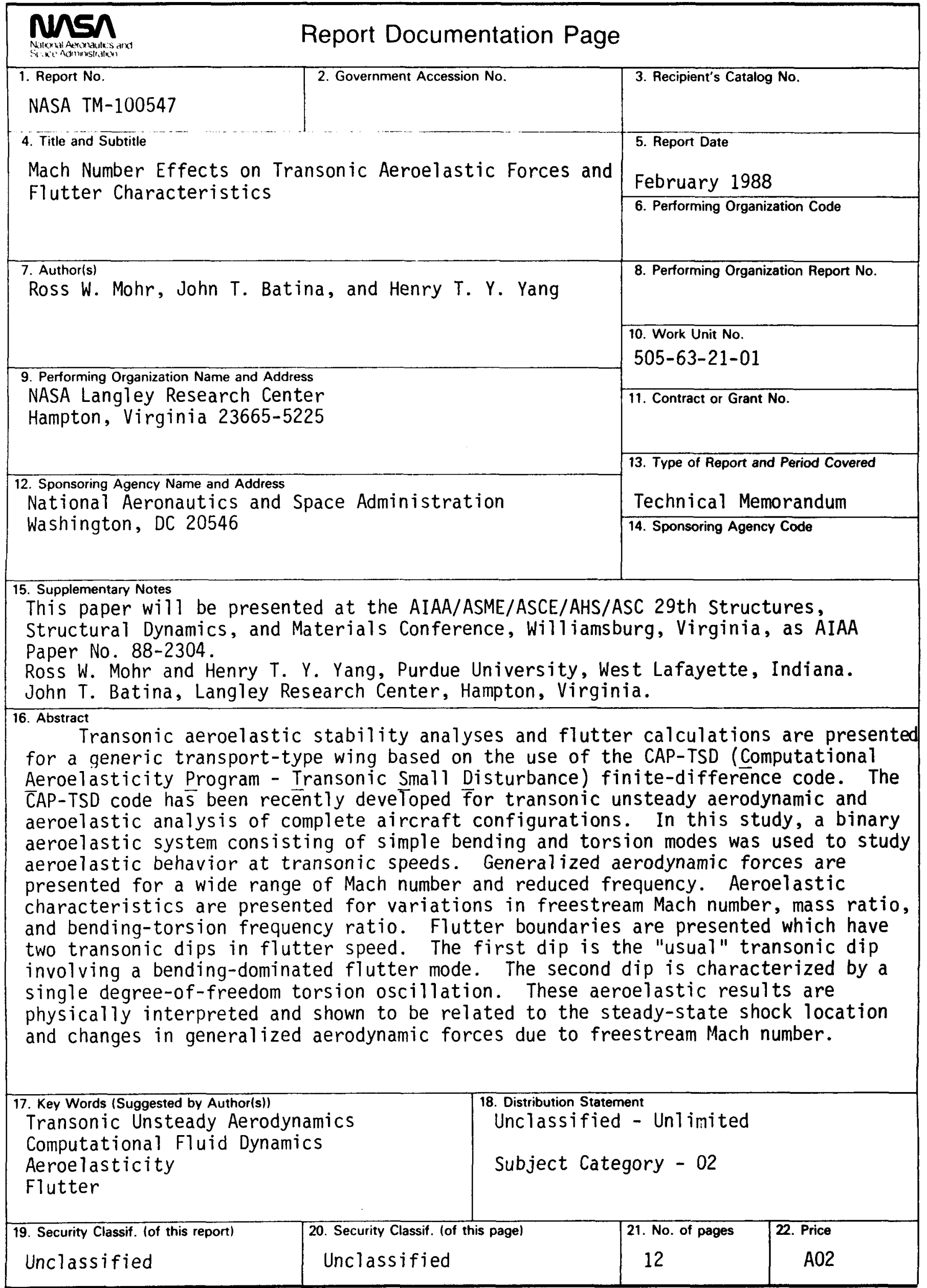

